\title{
A meta-analysis of tumor necrosis factor- $a-308$ G>A polymorphism in gastric cancer
}

\author{
Xin Jiang', Niyaz Ahmad Naikoo ${ }^{2, *}$, Shuowei Gao ${ }^{3}$
}

\section{Abstract}

Background: Gastric cancer (GC) is the common cause of cancer-related deaths worldwide and inflammation represents the early phases in the GC.

Objective: To review the tumor necrosis factor (TNF)- $\alpha-308 \mathrm{G}>\mathrm{A}$ (GG, GA, and AA) in GC by meta-analysis studies for any differences in TNF- $\alpha-308 \mathrm{G}>\mathrm{A}$ gene polymorphisms.

Methods: Case-control studies published from 2003 to 2017 were identified by searching PubMed, EMASE, and the Internet with the English language. The analysis published on TNF- $\alpha-308 \mathrm{G}>\mathrm{A}$ polymorphism was analyzed and a limited number of articles were included in the present study. TNF- $\alpha-308 \mathrm{G}>\mathrm{A}$ from 4,157 patients and 5,185 healthy controls was evaluated. Studies were evaluated using Cochrane $Q$-test and publication bias was evaluated by constructing funnel plots.

Results: Overall, TNF- $\alpha-308$ GA genotype showed significant association $[P<0.0001$, odds ratio (OR), 95\% confidence interval $(\mathrm{CI})=0.82(0.74-0.91)]$. However, meta-analysis of TNF- $\alpha-308$ genotypes (GG, GA, AA, and $\mathrm{GA}+\mathrm{AA})$ between $\mathrm{GC}$ patients and controls showed nonsignificant association with $\mathrm{GC}[P>0.05$, recessive model: $\mathrm{OR}=1.38,95 \%$ CI: 1.15-1.66; dominant model: $\mathrm{OR}=1.23,95 \%$ CI: $1.09-1.39 ;(\mathrm{G} / \mathrm{A})$ vs. $(\mathrm{G} / \mathrm{G}): \mathrm{OR}=1.15,95 \%$ CI: $1.02-1.28 ;(A / A)$ vs. $(\mathrm{G} / \mathrm{G})$ : OR $=1.44,95 \%$ CI: 1.19-1.73]. Analysis stratified by ethnicity showed same results in Asian and Caucasian populations.

Conclusions: Results revealed nonsignificant association of TNF- $\alpha-308$ genotypes (GG, GA, AA, and GA + AA) and GC. TNF- $\alpha-308$ GA genotype showed significant association whereas homozygous genotype AA did not show association with GC risk.

Keywords: gastric cancer; meta-analysis; TNF- $\alpha-308$

Gastric cancer (GC) is commonly diagnosed with malignancies and remained a considerable health problem [1]. Tumor necrosis factor-alpha (TNF- $\alpha$ ) produced by macrophages is a cytokine playing a pivotal role in the pathogenesis of malignant diseases [2]. TNF- $\alpha$ is a pro-inflammatory cytokine involved in the growth, differentiation, and survival of many cells. TNF- $\alpha$ is also playing an important role in the pathogenesis of cancer [3]. Studies have shown that TNF- $\alpha$ promoter polymorphism-308 (rs1800629) may regulate TNF- $\alpha$ production [4]. TNF- $\alpha-308$ has been confirmed as a risk factor

*Correspondence to: Niyaz Ahmad Naikoo, Department of Biotechnology, Government College for Women, Cluster University Srinagar, Jammu and Kashmir 190001, India, e-mail: drniyaz@gcwmaroad.edu.in

'Basic Medical Sciences, College of Jilin University, Changchun City, Jilin 130021, China

2Department of Biotechnology, Government College for Women, Cluster University Srinagar, Jammu 190001, India

${ }^{3}$ Department of Gastrointestinal Colorectal and Anal Surgery, China-Japan Union Hospital of Jilin University, Jilin 130033, China

¿ Open Access. @ 2020 Jiang et al., published by Sciendo. (c) Br-NC-ND This work is licensed under the Creative Commons Attribution NonCommercial-NoDerivatives 4.0 License. 
for a range of cancers such as breast, gastric, and hepatocellular cancers $[5,6]$. Several single-nucleotide polymorphisms have been identified in the promoter region of TNF- $\alpha$. TNF- $\alpha-308$ $\mathrm{G}>\mathrm{A}$ is a mediator of immune response and it shares many biological properties with interleukin 1 (IL-1). TNF- $\alpha-308$ A allele is an important candidate accounting for the increased risk of gastric carcinoma $[7,8]$. TNF- $\alpha-857 \mathrm{C}>\mathrm{T}$ is associated with higher transcriptional activity [9].

The number of TNF- $\alpha$ single nucleotide polymorphisms in promoter has been indicated to regulate TNF- $\alpha$ transcription $[10,11]$. TNF- $\alpha-308$ is an extensively studied single-nucleotide polymorphism in GCs [12-14]. The results on TNF- $\alpha-308$ G $>$ A have been inconsistent. Therefore, a meta-analysis was conducted to find out the association of TNF- $\alpha-308 \mathrm{G}>\mathrm{A}$ and GC.

\section{Method}

\section{Data extraction}

Pubmed and Google databases were used to extract all relevant literature on human studies, TNF- $\alpha$ polymorphism. Data from eligible studies were identified with respect to year of publication, first author name, country, source of sample, cases and controls, genotype frequencies, and reported associations. This study has a total of 12 articles on TNF- $\alpha-308$ $\mathrm{G}>\mathrm{A}$ and GC. Data on the author's last name, publication year, country of origin, study population source, genotypes, number of cases and controls, and TNF- $\alpha-308$ genotyping method were extracted. Data were procured independently from each study using a predefined form and conflicts were resolved by discussion as shown in Figure 1.

\section{Statistical analysis}

The genotype frequency and distribution among controls and cases were analyzed by Fisher's test. $P<0.05$ is considered statistically significant. Comprehensive meta-analysis V.3 employed for calculation of pooled odds ratios (ORs) and 95\% confidence interval (CI). The Cochran $Q$-test and index $\left(I^{2}\right)$ were used to assess the heterogeneity within studies. A $Q$-test with $P<0.10$ indicated significant heterogeneity. A fixed or random-effects model was used to calculate OR and $95 \%$ CIs based on heterogeneity strength. $I^{2}$ values range from $4 \%$ to $100 \%$, where lower values indicate nonsignificant heterogeneity and higher values indicate a high degree of heterogeneity. Publication bias was investigated by the construction of funnel plots and Egger's regression analysis.
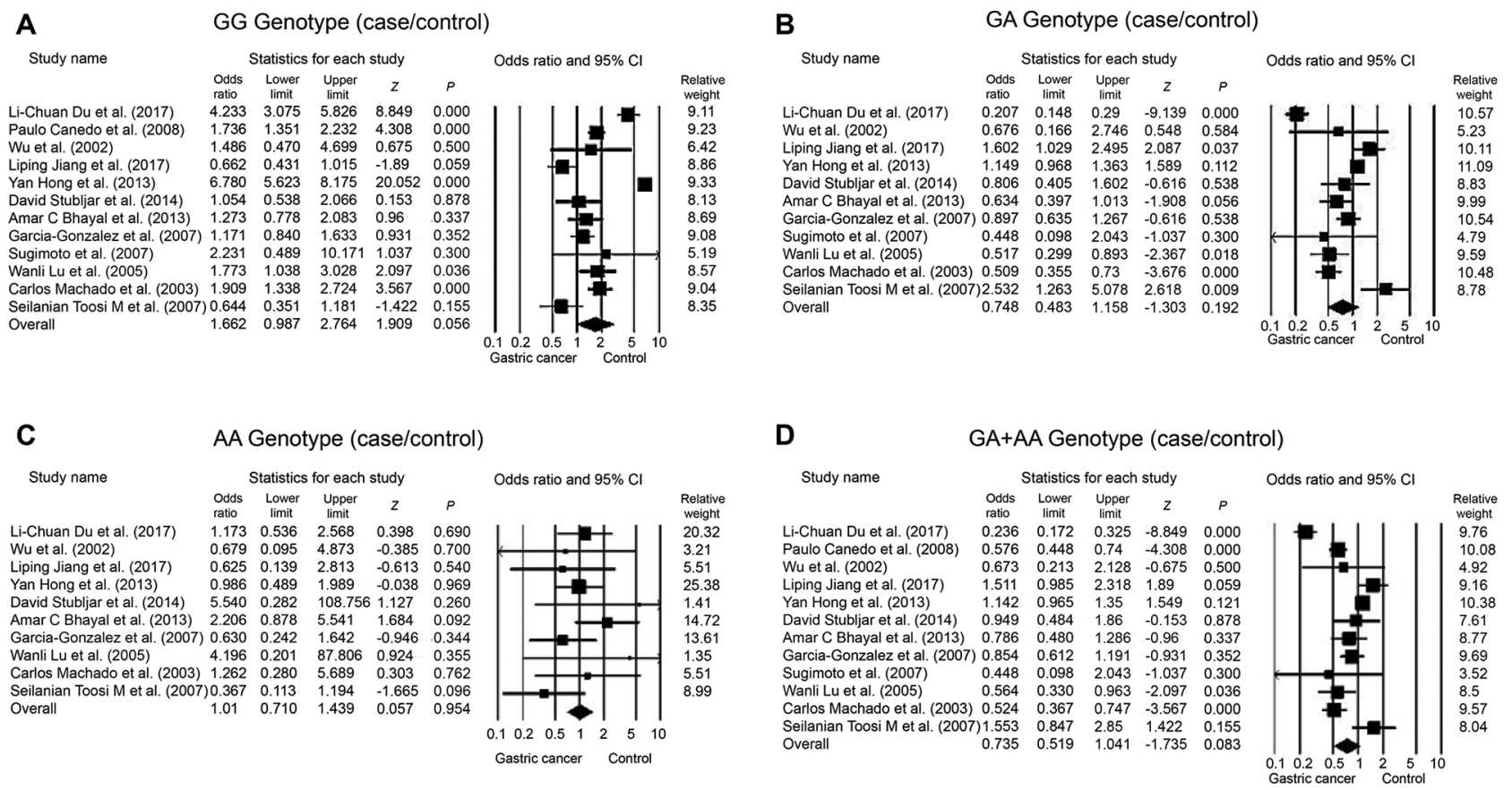

Figure 1. Flow chart of studies identification and inclusion. A. Forest plot of gastric cancer (GC) risk with the TNF- $\alpha$-308 GG genotype (overall). A random-effects model was used for calculation. B. Forest plot of GC risk with the TNF- $\alpha-308$ GA genotype (overall). A random-effects model was used for calculation. C. Forest plot of GC risk with the TNF- $\alpha$-308 AA genotype (overall). A fixed-effects model was used for calculation. D. Forest plot of GC risk with the TNF- $\alpha-308 \mathrm{GA}+$ AA genotype (overall). A random-effects model was used for calculation. 


\section{Results}

\section{Study characteristics}

Twelve studies were analyzed in which 12 sets of data were compared with distributions of TNF- $\alpha-308$ genotypes in patients and healthy controls.

Analysis using Fischer's exact test revealed that TNF$\alpha-308$ GA genotype was significantly prevalent in GC cases compared with healthy controls $[P<0.0001$, OR $(95 \% \mathrm{CI})=0.82(0.74-0.91)]$, and homozygous genotype AA did not show association with $\mathrm{GC}$ risk $[P=0.49$, OR $(95 \% \mathrm{CI})=0.62(0.44-0.86)]$, as shown in Table 1, thus indicating possible association of TNF- $\alpha-308$ GA with the GC susceptibility. However, a meta-analysis of TNF- $\alpha$ 308 genotypes in GC cases and healthy controls revealed nonsignificant association, hence we evaluated 54 articles; after the screening of titles, abstracts and analysis of articles, 12 eligible studies were screened for meta-analysis, including 4,852 GC patients and 5,197 controls. Characteristics of studies and other relevant data, such as genotype frequency in cases and controls, are shown in Table 2. $Q$-test and $I^{2}$ statistics showed heterogeneity in the included studies, and therefore a random-effects model was employed for genotype comparison; however, nonsignificant heterogeneity was observed in TNF- $\alpha-308$ AA genotype and therefore fixedeffects model was employed for genotype comparison among cases and controls (Table 3).

\section{TNF-a-308 GG distribution}

The TNF- $\alpha-308$ GG genotype distribution in patients and controls was $3,200 / 4,300$. Significant heterogeneity among studies with $Q=222.03, I^{2}=95.04 \%$, and $P=0.00$ is indicated

Table 1. Distribution of TNF- $\alpha-308$ G>A (rs1800629) genotypes in GC patients and healthy controls

\begin{tabular}{lcccc}
\hline TNF-a-308 G>A (rs1800629) Genotypes & GC & Healthy controls & OR (95\% CI) & P \\
\hline GG & 3,200 & 4,300 & Reference & - \\
GA & 891 & 806 & $0.82(0.74-0.91)$ & $<0.0001$ \\
AA & 66 & 79 & $0.62(0.44-0.86)$ & 0.49 \\
\hline
\end{tabular}

GG, homozygous wild; GA, heterozygous; AA, homozygous variant; TNF-a, tumor necrosis factor-alpha; GC, gastric cancer; OR, odds ratio; $\mathrm{Cl}$, confidence interval.

Table 2. Main characteristics of studies selected for meta-analysis and distribution of TNF- $\alpha-308 \mathrm{G}>\mathrm{A}$ (rs1800629) polymorphism in cases and healthy controls

\begin{tabular}{|c|c|c|c|c|c|c|c|}
\hline \multirow[t]{2}{*}{ First author (year) } & \multirow[t]{2}{*}{ Ethnicity/country } & \multicolumn{3}{|c|}{ Case } & \multicolumn{3}{|c|}{ Healthy control } \\
\hline & & GG & GA & AA & GG & $\overline{G A}$ & AA \\
\hline Li-Chuan Du et al. (2017) & China & 204 & 184 & 12 & 326 & 60 & 14 \\
\hline Paulo Canedo et al. (2008) & Portugal & 330 & $178^{*}$ & & 544 & $169^{*}$ & \\
\hline Wu et al. (2002) & USA & 144 & 4 & 2 & 214 & 4 & 2 \\
\hline Liping Jiang et al. (2017) & China & 207 & 30 & 3 & 411 & 95 & 4 \\
\hline Yan Hong et al. (2013) & China & 1335 & 333 & 18 & 1585 & 295 & 14 \\
\hline David Stubljar et al. (2014) & Slovenia & 63 & 20 & 0 & 83 & 22 & 3 \\
\hline Amar C Bhayal et al. (2013) & India & 32 & 76 & 6 & 76 & 128 & 25 \\
\hline Garcia-Gonzalez et al. (2007) & Spain & 309 & 84 & 11 & 320 & 77 & 7 \\
\hline Sugimoto et al. (2007) & Japan & 101 & 4 & 0 & 169 & 3 & 0 \\
\hline Wanli Lu et al. (2005) & China & 214 & 36 & 0 & 274 & 24 & 2 \\
\hline Carlos Machado et al. (2003) & Portugal & 179 & 105 & 3 & 231 & 69 & 4 \\
\hline Seilanian Toosi M et al. (2007) & Iran & 82 & 15 & 11 & 67 & 29 & 4 \\
\hline
\end{tabular}

${ }^{*}(\mathrm{GA}+\mathrm{AA})$. 
in Table 3. Meta-analysis by random-effects model indicated nonsignificant results with pooled OR $=1.65(0.98-2.76)$, $Z=1.9$, and $P=0.056$ as shown in Figure $1 \mathbf{A}$.

\section{TNF-a-308 GA distribution}

TNF- $\alpha-308$ GA genotype distribution in patients and controls was $891 / 806$ and significant heterogeneity with $Q=109.14$, $I^{2}=90.83 \%$, and $P=0.00$ (Table 3). Meta-analysis of the TNF- $\alpha-308$ GA genotype did not show significant results with pooled $\mathrm{OR}=0.74(0.48-1.15), Z=-1.30$, and $P=0.19$ using random-effects model as indicated in the forest plot in Figure 1B.

\section{TNF-a-308 AA distribution}

TNF- $\alpha-308$ AA genotype distribution in patients and controls was 66/79. Nonsignificant heterogeneity with $Q=9.39$, $I^{2}=4.23 \%$, and $P=0.40$ is shown in Table 3. The metaanalysis overall showed nonsignificant results with pooled $\mathrm{OR}=1.01(0.71-1.43), Z=0.57$, and $P=0.95$ by fixed-effects model.

\section{TNF-a-308 GA + AA distribution}

TNF- $\alpha-308$ GA + AA genotype distribution in patients and controls was $1,135 / 1,054$. Significant heterogeneity with $Q=100.59, I^{2}=89.06 \%$, and $P=0.00$ is shown in Table 3 . Meta-analysis showed nonsignificant results with pooled $\mathrm{OR}=0.73(0.51-1.04), Z=-1.73$, and $P=0.082$ by randomeffects model.

\section{Publication bias}

Begg's funnel plot and Egger's test were used to assess publication bias and results did not indicate evidence of publication bias for TNF- $\alpha-308$ polymorphisms in cases and controls [(TNF- $\alpha-308$ GG Begg's test, $P=0.84$; Egger's test, $P=0.39$ ), (TNF- $\alpha-308$ GA Begg's test, $P=1.0$; Egger's test, $P=0.95)$, (TNF- $\alpha-308$ AA Begg's test, $P=0.60$; Egger's test, $P=0.56$ ), and (TNF- $\alpha-308$ GA Begg's test, $P=1.0$; Egger's test, $P=0.95)]$ as shown in Figure 2.
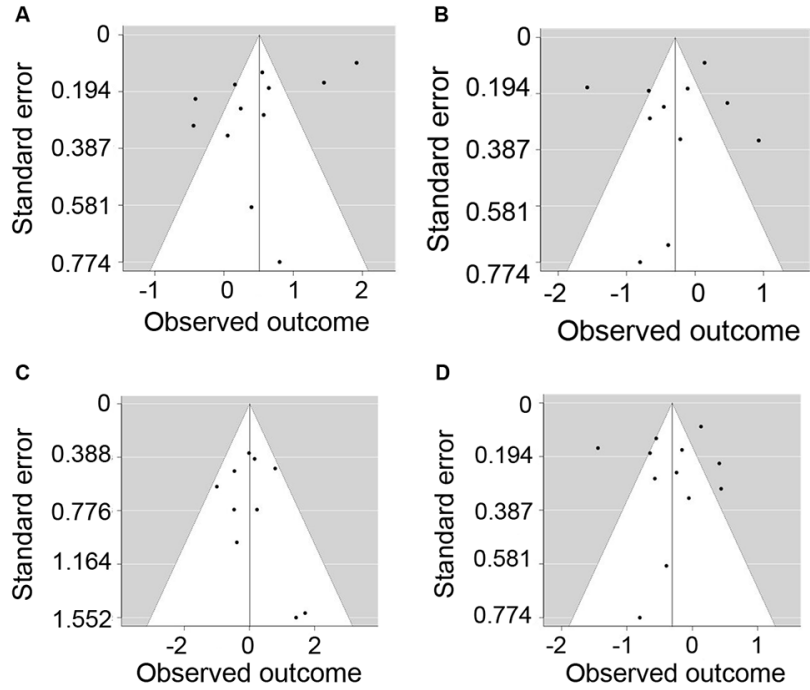

Figure 2. A. Begg's funnel plot for publication bias test for TNF- $\alpha-308$ GG polymorphism. No evidence of publication bias ( $P=0.84)$. B. Begg's funnel plot for publication bias test for TNF- $\alpha-308$ GA polymorphism. No evidence of publication bias $(P=1.0)$. C. Begg's funnel plot for publication bias test for TNF- $\alpha-308$ AA polymorphism. No evidence of publication bias $(P=0.60)$. D. Begg's funnel plot for publication bias test for TNF- $\alpha-308$ $\mathrm{GA}+\mathrm{AA}$ polymorphism. No evidence of publication bias $(P=1.0)$.

Table 3. Statistics to test publication bias and heterogeneity in the meta-analysis

\begin{tabular}{|c|c|c|c|c|c|c|c|c|c|c|}
\hline \multirow{2}{*}{$\begin{array}{l}\text { No. of } \\
\text { studies }\end{array}$} & \multirow{2}{*}{$\begin{array}{l}\text { Genotype } \\
\text { (case/control) }\end{array}$} & \multicolumn{2}{|c|}{ Egger's regression analysis } & \multicolumn{4}{|c|}{ Effect size and $95 \% \mathrm{Cl}$} & \multicolumn{3}{|c|}{ Heterogeneity analysis } \\
\hline & & Intercept & $P$ & RE-OR & $95 \% \mathrm{Cl}$ & $Z$ & $P$ & Q-Statistic & $P$ (heterogeneity) $\dagger$ & $I^{2}(\%) \ddagger$ \\
\hline 12 & GG $(3,200 / 4,300)$ & 0.508 & 0.39 & 1.65 & $(0.98-2.76)$ & 1.90 & 0.056 & 222.03 & 0.00 & 95.04 \\
\hline 11 & GA (891/806) & -0.291 & 0.95 & 0.74 & $(0.48-1.15)$ & -1.30 & 0.19 & 109.14 & 0.00 & 90.83 \\
\hline 10 & $A A^{*}(66 / 79)$ & 0.009 & 0.56 & 1.01 & $(0.71-1.43)$ & 0.57 & 0.95 & 9.39 & 0.40 & 4.23 \\
\hline 12 & $\begin{array}{l}\mathrm{GG}+\mathrm{GA} \\
(1,135 / 1,054)\end{array}$ & -0.308 & 0.95 & 0.73 & $(0.51-1.04)$ & -1.73 & 0.082 & 100.59 & 0.00 & 89.06 \\
\hline
\end{tabular}

Chi-square Q-statistic for homogeneity in random-effects model.

*Fixed-effects model.

† P for the Q-statistic in random-effects model.

¥ Higgins $I^{2}$ statistic for heterogeneity in random-effects model.

$\mathrm{Cl}$, confidence interval; $\mathrm{RE}$, random effect; $\mathrm{OR}$, odds ratio. 


\section{Discussion}

Inflammation is considered as one of the key factors involving in the pathogenesis of cancer, and TNF- $\alpha$ is believed to be one of the most crucial inflammatory cytokines. TNF- $\alpha$ $\mathrm{G}>\mathrm{A}$ is produced by macrophages, neutrophils, fibroblasts, T-cells, B-cells, and tumor cells, and it has been reported to play an important role in the pathogenesis of cancer $[3,15]$. The transcription of TNF- $\alpha$ is regulated under genetic control. Studies have indicated that promoter polymorphisms at rs361525, rs1800629, rs1799724, and rs1799964 may regulate TNF- $\alpha$ production and it was reported that expression level of TNF- $\alpha$ was proved to be affected by polymorphisms in the promoter region of TNF [4, 16, 17]. Results of metaanalysis showed an overall nonsignificant association of TNF- $\alpha-308$ GG, GA, AA, and GA + AA genotypes with GC. The discrepancy in results could be identified in sample size, ethnicity, or etiological factors that contribute to the heterogeneity.

Several meta-analyses on TNF- $\alpha-308 \mathrm{G}>$ A polymorphism showed an association of TNF- $\alpha-308 \mathrm{G}>$ A genotypes with GC risk $[18,19]$. Overall results on TNF- $\alpha-308 \mathrm{G}>\mathrm{A}$ genotypes did not show the association between AA genotype and GC risk using GG as the reference genotype yielded statistically significant risk of GC with TNF- $\alpha-308$ GA genotype. However, the meta-analysis was conducted using fixedand random-effects models; none of the TNF- $\alpha-308 \mathrm{G}>\mathrm{A}$ genotypes showed significant association with GC. Earlier studies suggested that frequencies of genetic markers often show high variations among various ethnic and racial groups whereas differences in genetic effects in terms of ORs are much less common [20]. Heterogeneity between studies was high for TNF- $\alpha-308$ genotypes and low for TNF- $\alpha-308$ AA genotype, as indicated by the $I^{2}$ value of $>80$. Studies were subjected to publication bias using funnel plots and Egger's weighted regression and rank correlation method [21], and no publication bias was detected in studies. The summary ORs for GG, GA, AA, and GA + AA genotypes remained close to null and were nonsignificant. However, the hypothesis that TNF- $\alpha$ genotypes may be associated with GC is still unclear and the results of earlier association studies have largely been inconsistent. The present study supports the fact that TNF- $\alpha-308 \mathrm{G}>\mathrm{A}$ genotypes have no role in early diagnosis and treatment of gastric inflammation. Since TNF- $\alpha-308$ genotypes do not have a role in GC predisposition, TNF$\alpha-308 \mathrm{G}>\mathrm{A}$ genotypes make nonsignificant contribution to prevent the occurrence of GC.

To understand the carcinogenesis of GC, prospective studies in combination with the analysis of other cytokines are required. Earlier identification of TNF- $\alpha$ polymorphisms yielded some suggestions on understanding the genetic predisposition of gastric and colorectal cancers [22].

Taken together, meta-analysis data do not support the association between TNF- $\alpha-308$ genotypes (GG, GA, AA, and GA + AA) and GC predisposition. However, additional case-control studies in different populations are still needed.

Author contributions. All the authors contributed substantially to the study conception and design, acquisition of data, and its analysis. XJ and NN drafted the manuscript. SG contributed substantially to its critical revision. All the authors approved the final version submitted for publication and take responsibility for the statements made in the published article.

Acknowledgments. This study received funding from Jilin Province Science and Technology Development Project (No. 20170414034GH) and China Postdoctoral Science Foundation Funded Projects (No. 2018M63186). The authors are grateful to the Department of Biotechnology and Bioinformatics, Government College for Women, for their encouragement in compiling the data on gastric cancer.

Conflict of interest statement. The authors have completed and submitted the ICMJE Uniform Disclosure Form for Potential Conflicts of Interest. None of the authors disclose any conflict of interest.

Data sharing statement. No data sets were generated or analyzed during the present study. The present review is based on the references cited.

\section{References}

[1] Parkin DM, Bray F, Ferlay J, Pisani P. Global cancer statistics; 2002. CA Cancer J Clin. 2005; 55:74-108.

[2] Bazzoni F, Beutler B. The tumor necrosis factor ligand and receptor families. N Engl J Med. 1996; 334:1717-25.

[3] Wang SS, Purdue MP, Cerhan JR, Zheng T, Menashe I, Armstrong $\mathrm{BK}$, et al. Common gene variants in the tumor necrosis factor (TNF) and TNF receptor superfamilies and NF-kB transcription factors and non-Hodgkin lymphoma risk. PLoS One. 2009; 4:e5360.

[4] Hellmig S, Fischbach W, Goebeler-Kolve ME, Folsch UR, Hampe J, Schreiber S. A functional promotor polymorphism of TNF-alpha is associated with primary gastric B-Cell lymphoma. Am J Gastroenterol. 2005; 100:2644-9.

[5] Lu P-H, Tang Y, Li C, Shen W, Ji L, Guo Y-J, Tao G.-Q. Meta-analysis of association of tumor necrosis factor alpha-308 gene promoter polymorphism with gastric cancer. Zhonghua Yu Fang Yi Xue Za Zhi. 2010; 44:209-14.

[6] Shen C, Sun H, Sun D, Xu L, Zhang X, Liu A, et al. Polymorphisms of tumor necrosis factor-alpha and breast cancer risk: a meta-analysis. Breast Cancer Res Treat. 2011; 126:763-70. 


\section{Allli ASIAN BIOMEDICINE}

[7] Gorouhi F, Islami F, Bahrami H, Kamangar F. Tumour-necrosis factor-A polymorphisms and gastric cancer risk: a meta-analysis. Br J Cancer. 2008; 98:1443-51.

[8] Zhang J, Dou C, Song Y, Ji C, Gu S, Xie Y, Mao Y. Polymorphisms of tumor necrosis factor-alpha are associated with increased susceptibility to gastric cancer: a meta-analysis. J Hum Genet. 2008; 53:479-89.

[9] Hohjoh H, Tokunaga K. Allele-specific binding of the ubiquitous transcription factor OCT-1 to the functional single nucleotide polymorphism (SNP) sites in the tumor necrosis factor-alpha gene (TNFA) promoter. Genes Immun. 2001; 2:105-9.

[10] Dunham I, Sargent CA, Trowsdale J, Campbell RD. Molecular mapping of the human major histocompatibility complex by pulsed-field gel electrophoresis. Proc Natl Acad Sci. 1987; 84:7237-41.

[11] Wilson AG, Symons JA, McDowell TL, McDevitt HO, Duff GW. Effects of a polymorphism in the human tumor necrosis factor alpha promoter on transcriptional activation. Proc Natl Acad Sci. 1997; 94:3195-9.

[12] El-Omar EM, Rabkin CS, Gammon MD, Vaughan TL, Risch HA, Schoenberg JB, et al. Increased risk of noncardia gastric cancer associated with proinflammatory cytokine gene polymorphisms. Gastroenterology. 2003; 124:1193-201.

[13] Canedo P, Durães C, Pereira F, Regalo G, Lunet N, Barros H, et al. Tumor necrosis factor alpha extended haplotypes and risk of gastric carcinoma. Cancer Epidemiol Biomarkers Prev. 2008; 17:2416-20.

[14] Burada F, Angelescu C, Mitrut P, Ciurea T, Cruce M, Saftoiu A, et al. Interleukin-4 receptor $-3223 \mathrm{~T}$ and $8594 \mathrm{C}$ polymorphism is associated with increased gastric adenocarcinoma risk. Can J Gastroenterol. 2012; 26:532-36.

[15] Anderson GM, Nakada MT, DeWitte M. Tumor necrosis factor-alpha in the pathogenesis and treatment of cancer. Curr Opin Pharmacol. 2004; 4:314-20.

[16] Higuchi T, Seki N, Kamizono S, Yamada A, Kimura A, Kato H, Itoh K. Polymorphism of the 5'-flanking region of the human tumor necrosis factor (TNF)-alpha gene in Japanese. Tissue Antigens. 1998; 51:605-12.

[17] Lindholm E, Bakhtadze E, Cilio C, Agardh E, Groop L, Agardh CD. Association between LTA; TNF and AGER polymorphisms and late diabetic complications. PLoS One. 2008; 3:e2546.

[18] Rokkas T, Sechopoulos P, Pistiolas D, Kothonas F, Margantinis G, Koukoulis G. Population differences concerning TNF-alpha gene polymorphisms in gastric carcinogenesis based on meta-analysis. Ann Gastroenterol. 2014; 27:139-48.

[19] Du LC, Gao R. Role of TNF-alpha -308G/A gene polymorphism in gastric cancer risk: a case control study and meta-analysis. Turk J Gastroenterol. 2017; 28:272-82.

[20] Ioannidis JP, Ntzani EE, Trikalinos TA. 'Racial' differences in genetic effects for complex diseases. Nat Genet. 2004; 36:1312-8.

[21] Begg CB, Mazumdar M. Operating characteristics of a rank correlation test for publication bias. Biometrics. 1994; 50: 1088-101.

[22] Campbell DA, Field M, McArdle CS, Cooke TG, Gallagher G. Polymorphism at the tumour necrosis factor locus: a marker of genetic predisposition to colorectal cancer? Lancet. 1996; 347:1706. 